\title{
7. SUPERNOVAE
}

\section{Scientific Organizing Committe}

$$
\text { V. TRIMBLE (Chairman) }
$$

G.S. BISNOVATYI-KOGAN, R. CHEVAL IER, S. HAYAKAWA, T. LU, N. PANAGIA, S. RAMADURAI, G. SETTI, F.G. SMITH, D. SUGIMOTO, J. TRUEMPER, A.V. TUTUKOV, S. van DEN BERGH, B. WARNER, J.C. WHEELER

Supporting Commissions : $27,28,34,35$, $40,44,47,48$ 


\author{
Sidney van den Bergh \\ Dominion Astrophysical Observatory \\ Herzberg Institute of Astrophysics \\ 5071 West Saanich Road \\ Victoria, B.C. Canada, V8X 4M6
}

ABSTRACT. Observations by Hartwig, Wolf, Duner and Bigourdan are used to show that $S N 1885$ a reached $\mathrm{M}_{\mathrm{V}}(\max )=-18.8 \pm 0.5$ on 17 or 18 August 1885 . The lightcurve of $S$ And is found to have declined more rapidly after maximum than that of any other well-observed supernova. The existence of $S$ And and the relatively young age of the supernova remnant Sgr A East appear to indicate that the supernova frequencies near the nucleus of $M 31$ and the Galactic centre are respectively $\sim 10^{2}$ and $\sim 10^{3}$ times higher than expected. It is spectulated that $S$ And may belong to a population of supernovae ("type 0") that only occur near the nuclei of giant galaxies. Beyond the Local Group such objects might perhaps have been missed because of their fast decline and close proximity to luminous nuclei.

\title{
I. INTRODUCTION
}

The supernova $S$ Andromedae was discovered by Ernst Hartwig at the Dorpat Observatory on 20 August 1885. A very detailed description of its discovery is given by Jones (1976); a more succinct account is provided by Gaposchkin (1961). Flin et al. (1979) give a very complete bibliography of SN 1885a. Not included in this bibliography are papers on unsuccessful attempts by Spencer and Burke (1973) and by Dickel and D'Odorico (1984) to detect radio emission from $S$ And and a search for an optical pulsar by Middleditch and Kristian (1984) who find that any source with a frequency in the range 0 to $500 \mathrm{~Hz}$ at the position of SN 1885 a must have in $>21.5$.

\section{THE LIGHTCURVE}

Early photometric observations of $S$ And are critically discussed by Gaposchkin (1961), who includes corrections to take into account systematic errors in the magnitudes of neighboring Bonner Durchmusterung stars which Hartwig and Wolf used as standards for their visual brightness estimates of the supernova. These observations out line the supernova lightcurve between 16 August and 3 September 1885. Recently de Vaucouleurs has rediscovered valuable series of observations of $S$ And by N.C. Dunér of the Lund Observatory (de Vaucouleurs, Hansson and Lyngà 
1985) by G. Bigourdan of the Paris Observatory. In order to place these observations on a modern photometric system de Vaucouleurs and Buta (1981) have reobserved the comparison stars used by Bigourdan on the UBV system. The supernova lightcurve reconstructed from the early observations by Hartwig and Wolf and from the later data of Bigourdan and Duner is plotted in Fig. 1. The scatter is much reduced from that in plots (e.g. Jones 1976) which show all published magnitude estimates of $S$ And. Figure 1 shows that $S N 1885$ a was fainter than $V=8$ on 16 August and had $\mathrm{V}<6$ on 20 August 1885. Subsequently the supernova declined monotonically in brightness. In late August the rate of decline was $\sim 0.12$ mag day ${ }^{-1}$. By late October 1885 this had decreased to $\sim 0.04$ mag day-1.

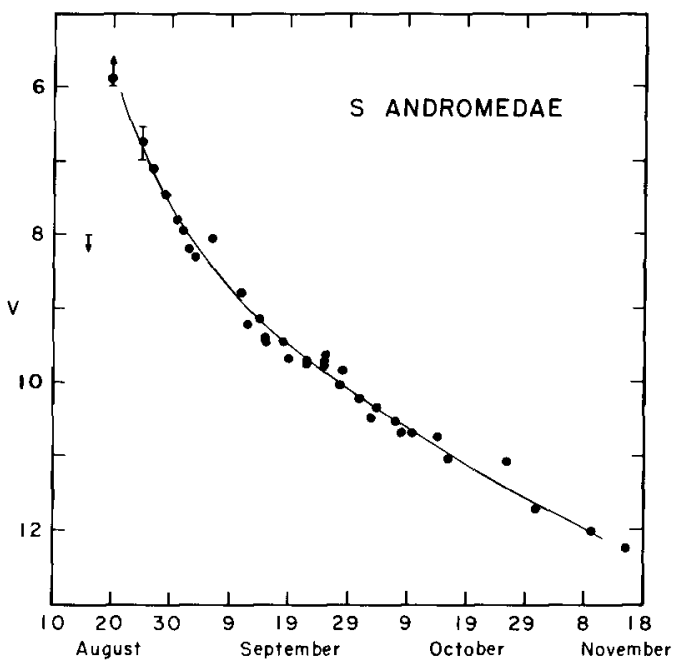

Fig. 1. Lightcurve of $S$ And based on observations by Hartwig and Wolf prior to 5 September 1885 and by Duner and Bigourdan after that date. The lightcurve suggests that $\mathrm{SN} 1885 \mathrm{e}$ reached $\mathrm{V}(\max )=$ $5.5 \pm 0.5$ on 17 or 18 August 1885 .

Inspection of $\mathrm{Fig} \cdot 1$ suggests that $\mathrm{S}$ And reached $\mathrm{V}(\max )=$ $5.5 \pm 0.5$ (estimated exror) on 17 August or 18 August 1885. Adopting an M31 distance modulus $(m-M)_{V}=24.32 \pm 0.11$ (van den Bergh 1977) then yields $M_{V}(\max )=-18.8 \pm 0.5$ for $S N 1885 \mathrm{a}$. Since the nuclear bulge of M31 is relatively dust-free absorption probably does not add a major contribution to the uncertainty of this result.

Among supernovae with photoelectrically measured lightcurves in $\mathrm{V}$ (Younger and van den Bérgh 1985) none provides anything resembing a close fit to the lightcurve of $S$ And. A similar conclusion was previously reached by Minkowski (1962) on the basis of less complete observations of the lightcurve of $S$ And and photographic lightcurves of supernovae beyond the Local Group.

Supernovae of type I exhibit their steepest decline 20 days after maximum 1ight. For 8 reasonably well-observed SNI (Younger and van den Bergh 1985) the mean rate of decline at 20 days past maximum light is $0.065 \pm 0.007 \mathrm{mag}$ day $^{-1}$ compared to a decline of $0.10 \pm 0.01$ (estimated 
error) mag day-1 for $S$ And. The four best-observed supernovae of type II have a rate of decline of only $0.026 \pm 0.009$ mag day ${ }^{-1}$ at 20 days past maximum. Such low rates of decline for SNII are clearly inconsistent with that observed in S And. It therefore appears unlikely that SN 1885a was, as has recent1y been suggested by Chugaj (1983), a fast-declining supernova of type II. Another argument against this hypothesis is that SNII are associated with a spiral-arm population (e.g. Maza and van den Bergh 1976), whereas S And occurred in the nuclear bulge of M31.

Between 30 and 80 days after maximum light a reasonable fit can be made between the SN 1885 a light curve and that of the we11-observed type I supernova $1972 \mathrm{e}$ in NGC 5253. Such a fit would, however, require NGC 5253 (which is a member of the NGC $5128 /$ NGC5236 cluster) to have a distance modulus $(m-M)_{V}=24.2$, which is clearly unreasonable! It follows that $S$ And was unusually faint well after maximum, rather than unusually bright near maximum light.

It is concluded that $S$ And was a most unusual object with a lightcurve that differed significantly from those of all supernovae that have so far been observed photoelectrically.

\section{THE POSITION OF S ANDROMEDAE}

According to de Vaucouleurs (1985), which supersedes de Vaucouleurs and Buta (1981), the position (epoch 1950) of the nucleus of M31 is:

$\alpha=0^{h} 40^{\mathrm{m}} 00.13 \delta=+40^{\circ} 59^{\prime} 42: 7$. According to his measurements SN 1885a occurred $15: 4 \mathrm{~W}$ and $3: 9 \mathrm{~S}$ of the nucleus. For an assumed distance of $630 \mathrm{kpc}$ (van den Bergh 1977) the projected linear separation of $\mathrm{S}$ And and the nucleus of M31 is only $49 \mathrm{pc}$.

If the Andromeda nebula were to be moved out to the distance of the Virgo cluster then the supernova would have occurred at a distance of only 0:5 from the nucleus! Since plates taken for supernova search programmes usually over-expose the central regions of galaxies it is quite conceivable that a hypothetical class of supernovae of "type 0 ", that only occur in or near the nuclei of giant galaxies, would have escaped detection. The rapid decline of objects similar to $S$ And would also militate against their discovery. SN 1885a decreased in brightness by 2 mag in $\sim 12$ days, whereas a typical supernova of type $I$ requires $\sim 40$ days to fall 2 mag below maximum.

\section{SUPERNOVA STATISTICS}

The region within $16^{\prime \prime}$ of the nucleus of M31 contains only $0.6 \%$ of the total luminosity of the Andromeda nebula. From an a priori point of view it is therefore highly improbable that the only supernova ever to have been observed in M31 should have occurred so close to the nucleus of this galaxy (cf. van den Bergh 1983a).

The area within 16" of the nucleus has $M_{B}=-14.3$, corresponding to $8.3 \times 10^{7} L_{0}$. If one supernova occurs within this region each century 
then the corresponding supernova rate is $\sim 120 \mathrm{SN}$ per century per $10^{10} \mathrm{~L}_{\mathrm{O}}$. This value is two orders of magnitude higher than the supernova rates of 0.24 and $1.18 \mathrm{SN}$ per century per $1010 \mathrm{~L}$, for $\mathrm{E}$ and $\mathrm{Sb}$ galaxies respectively, derived by Oemler and Tinsley (1979). Even allowing for the wel1-known vagaries of a posteriori statistics it seems that there might possibly be some grounds for the suspicion that $S$ And belongs to a class of nuclear supernovae which have so far eluded detection in more distance galaxies.

Some support for this hypothesis is provided by observations (Ekers et a1. 1983) of the supernova remnant Sgr A East; the centre of which is Iocated at a projected distance of $\sim 40^{\prime \prime}(1.9 \mathrm{pc})$ from the Galactic centre. According to Dort $(1977)$ the mass within 2.5 pc of the Galactic nucleus amounts to $1.3 \times 10^{7} \mathrm{M}_{\odot}$ i.e. $\sim 1 \times 10^{-4}$ of the total (non-halo) mass of the entire Milky Way system. Goss et al. (1983) estimate the age of $\mathrm{Sgr}$ A East to fall within the range $140 \overline{\text { to }} \overline{440}$ yrs. According to van den Bergh (1983b) the Galactic supernova rate is $\sim 2$ per century. The a priori probability that a supernova would occur within $2.5 \mathrm{pc}$ of the Galactic centre within the last 500 years is therefore $\sim 2 \times 10^{-4} \times 5=$ $1 \times 10^{-3}$. The supernova rate near the Galactic nucleus that is indicated by Sgr A East appears to be three orders of magnitude higher than expected. Both the observation of $\mathrm{S}$ And in $\mathrm{M} 31$ and of Sgr A East in the Galaxy therefore support the ad hoc hypothesis that the supernova frequency is unexpectedly high near the nuclei of giant spirals.

\section{DISCUSSION}

Three lines of evidence indicate that the nuclear regions of giant galaxies might produce a type of supernova that is not observed in the outer parts of galaxies: (1) The shape of the lightcurve of $S$ And appears to be unique - indicating that this object was neither an SNI nor an SNII, (2) the existence of $S$ And suggests that the supernova frequency close to the nucleus of $M 31$ is $\sim 10^{2}$ times higher than expected and (3) the existence and age of Sgr A East indicate a supernova frequency near the Galactic centre that is $\sim 10^{3}$ times higher than the expected combined frequency of supernovae of types I and II. A major problem with such high supernova rates is, however, that they would require most evolving stars in the nuclear regions of giant galaxies to become supernovae.

Possibly conventional supernova search techniques have missed fast-declining supernovae such as $S$ And that only occur very close to the nuclei of luminous galaxies. Some speculations on the nature of such a population of nuclear supernovae are given by van den Bergh (1983a). Perhaps ejecta from nuclear supernovae are, at least in part, responsible for the extreme strong-line characteristics (e.g. Mcclure 1969) of the innermost core of $\mathrm{M} 31$.

I am indebted to Dr. de Vaucouleurs for providing me with a recently re-determined position for $S$ Andromedae and for comments on an early draft of this paper. 
Chugaj, N.N.: 1983, Astron. Circ. USSR No. 1263. de Vaucouleurs, G.: 1985, private communication. de Vaucouleurs, G., Buta, R.: 1981, Astron.J. 93, 294.

de Vaucouleurs, G., Hansson, N., Lynğ, G.: 1985, Publ.A.S.P. 97, 30.

Dicke1, J.R., D'Odorico, S.: 1984, Monthly Notices Roy. Astron. Soc. 206, 351 .

Ekers, R.D., van Gorkum, J.H., Schwarz, U.J., Goss, W.M.: 1983, Astron. Astrophys. 122, 143.

F1in, P., Karpowicz, M., Murawski, W., Rudnicki, K.: 1979, Acta Cosmologica 8,87.

Gaposchkin, S.: 1961, Sky and Telescope 21, 326.

Goss, W.M., Schwarz, U.J., Ekers, R.D., van Gorkum, J.H.: 1983, in Supernova Remnants and Their X-Ray Emission, IAU Symposium No. 101, eds. J. Danziger and P. Gorenstein, Reidel, Dordrecht, p. 65. Jones, K.G.: 1976, J. History Astron. 7, 27.

Maza, J., van den Bergh, S.: 1976, Astrophys. J. 204, 519.

McClure, R.D.: 1969, Astron. J. 74, 50 .

Middleditch, J., Kristian, J.: 1984, Astrophys. J. 279, 157.

Oemler, A., Tinsley, B.M.: 1979, Astron. J. 84, 985.

Oort, J.H.: 1977, Ann. Rev. Astron. Astrophys. 15, 295.

Spencer, J.H., Burke, B.F.: 1973, Astrophys. J. (Letters) 185, L83.

van den Bergh, S.: 1977, in Decalages vers le Rouge et Expansion de

1'Univers, IAU Colloquium No. 37, eds. C. Balkowks1 and B.E.

Westerlund, CNRS, Paris), p. 14 .

van den Bergh, S.: 1983a, Astrophys. J. 265, 719.

van den Bergh, S.: 1983b, Publ.A.S.P. 95, 388.

Younger, P.F., van den Bergh, S.: 1985; Astron.Ap.Supp1. (in press). 